\title{
Pre-illness dietary factors in inflammatory bowel disease
}

\author{
S Reif, I Klein, F Lubin, M Farbstein, A Hallak, T Gilat
}

Department of Gastroenterology, Tel Aviv Sourasky Medical Center, Tel Aviv, Israel

I Klein

A Hallak

T Gilat

Department of Pediatric Gastroenterology, Tel Aviv Sourasky Medical Center,
Tel Aviv, Israel $S$ Reif

Sackler Faculty of Medicine, Tel Aviv University, Tel Aviv, Israel

F Lubin

M Farbstein

Correspondence to: Dr T Gilat,

Department of

Gastroenterology,

6 Weizman Street

Tel Aviv 64239, Israel.

Accepted for publication 20 February 1997

\begin{abstract}
Background-The effect of environmental factors has been demonstrated in the pathogenesis of inflammatory bowel disease (IBD). Nutrition may be one of them.

Aim-To investigate the pre-illness diet in patients with recent IBD in comparison with matched population and clinic controls.

Methods-Quantified dietary histories were obtained from 87 patients with recent IBD (54 ulcerative colitis (UC) and 33 Crohn's disease (CD)) and 144 controls. Odds ratios (OR) for IBD were derived for intake levels of various foods.

Results-A high sucrose consumption was associated with an increased risk for IBD (OR 2.85 ( $p=0.03)$ against population controls and $5 \cdot 3(p=0 \cdot 00)$ against clinic controls). Lactose consumption showed no effect while fructose intake was negatively associated with risk for IBD (NS). Similar trends were noted in UC and CD. A high fat intake was associated with an increased risk for UC; this was particularly marked for animal fat (OR 4.09, $p=0.02)$ and cholesterol (OR 4.57, $p=0 \cdot 02$ ). A high intake of fluids $(p=0 \cdot 04)$, magnesium ( $p=0 \cdot 04)$, vitamin C, and fruits (NS) was negatively associated with the risk for IBD, while a positive association was found for retinol $(p=0 \cdot 01)$. Most of the findings were similar in UC and CD except for potassium and vegetable consumption which showed a negative association only with risk for CD.

Conclusions-An association was found between pre-illness diet and subsequent development of UC and CD. The effect of dietary components may be primary or modulatory.

(Gut 1997; 40: 754-760)
\end{abstract}

Keywords: diet, nutrition, ulcerative colitis, Crohn's disease, inflammatory bowel disease.

The aetiology and pathogenesis of inflammatory bowel disease (IBD) have not yet been elucidated. Although the presence of genetic factors has been established by twin and family studies and genetic linkage analyses, ${ }^{1-4}$ environmental factors also play a significant role. ${ }^{5}$ In this context, it has been suggested that dietary factors may be important. ${ }^{67}$

The main evidence for the effect of environmental factors in IBD comes from epidemio- logical studies. The incidence of IBD rose very sharply in developing populations adopting an industrialised urban style of life. ${ }^{5}$ In developed populations, the incidence of Crohn's disease (CD) is higher in an urban environment than in the surrounding rural area. ${ }^{5}$ In developed urban populations, the incidence of $C D$ has risen by hundreds of percentages in recent decades. ${ }^{5}$ In all the above studies, the population, that is, the genetic factor, remained stable while the rapid changes in incidence indicate the effect of environmental factors.

Smoking has been shown to be a factor in the development of ulcerative colitis (UC) and CD. ${ }^{8-10}$ Diet has been a major suspect in the list of the as yet unidentified environmental factors. Patients with IBD, particularly CD, have been shown to consume more sucrose and refined carbohydrates and less fruit and vegetables compared with controls. ${ }^{11-14}$ Many of these studies did not differentiate between changes in diet due to disease and those antedating disease. ${ }^{14-17}$ Only a few studies examined the pre-illness diet in IBD and they reported conflicting results. ${ }^{11-13}{ }^{18-20}$ In this investigation, we studied the pre-illness diet of patients with recent onset of the disease and compared it with that of matched population and clinic controls.

\section{Methods}

POPULATION

Newly diagnosed patients in central Israel with definite UC and CD were interviewed as soon as possible after diagnosis during the years 1992-93. Patients were referred by various gastroenterologists in the study area. Not all recently diagnosed patients could be studied, but no referral bias could be identified, and the patients can be considered a random sample. All patients in the study were interviewed, after informed consent, within one year from onset of symptoms, most of them within three to four months from onset of symptoms. Two controls, one "healthy" population control, and one outpatient clinic control, were sought for each patient. Controls were matched by sex, age ( \pm 5 years), country of origin, and residential neighbourhood (reflecting socioeconomic status). For each patient, four potential population controls matched with the index patient by year of birth, ethnic origin, and area of residence were randomly selected from the Israel Population Registry. They were contacted by telephone, and the first one found to be suitably matched was personally interviewed. 
The second matched control was selected from the outpatient orthopaedic or ophthalmic clinics of the hospitals where the index cases were diagnosed. These were patients with a mild and usually transient illness that did not affect their diet or habits. Patients with any type of malignant disease or any known chronic illness were excluded. Two matched controls were obtained for $83 \%$ of patients. Due to the rigid matching criteria, only one matched control could be found in $17 \%$ of patients.

\section{QUESTIONNAIRE}

The collected data included information on diet composition and habits, physical activity, height, weight (at age 18 years and at present), lifetime smoking habits, alcohol intake, vitamin supplementation, and medications used.

One trained dietician conducted all standardised interviews lasting approximately one and a half hours, using a precoded questionnaire on diet and other lifestyle habits. The quantified dietary history questionnaire included 180 food items, among them ethnic foods and alcohol. Subjects were asked to describe the frequency and number of portions consumed and the usual portion size for each food item. Portion sizes, as determined by lifesize coloured photographs, were used to quantify nutrient intake as well as natural units and standard packing units.

Long term nutritional habits before the illness and symptoms began were evaluated, in addition to more recent diet and habits. For each item, the interviewees were asked if any changes had occurred in the consumption habits in the past five years because of illness or other reasons. If the answer was positive, first the more recent and then the past eating habits were ascertained for each item as well as the number of years since the change occurred. These were noted in two parallel sets of columns with identical sets of questions (frequency, number of portions, and portion size). This method allowed evaluation of habits before the illness began, in the same detail as for the more recent ones. Patients and controls were questioned in an identical manner as to changes in diet.

The reliability of our dietary history questionnaire has been evaluated in previous epidemiological studies on nutrition and cancer. This method was developed in the Department of Clinical Epidemiology of the Chaim Sheba Medical Center, Israel, and has been used previously in many nutrition epidemiological studies. $^{21-25}$

\section{DATA ANALYSIS}

Reported food consumption (monthly and weekly) was converted into average daily amounts. A comprehensive computer program was developed at the Epidemiology Department, Chaim Sheba Medical Center, based on information from international and local sources enabling quantitative analysis of the dietary profile at any level desired, including food items, food groups, and food components. ${ }^{26} 27$ First it converted all frequencies into weekly frequencies and to total weekly amounts, and then to mean daily consumption. For fruits and vegetables that are seasonable, length of season was taken into account when calculating average daily consumption.

Based on the distribution of the combined population of cases plus controls, three levels of intake (low, median, and high) were determined. Conditional logistic analysis adjusting for energy intake was performed. ${ }^{28}$ Odds ratios for each level as compared with the lowest one were evaluated, corresponding $95 \%$ confidence intervals were derived, and significance for linear trend was estimated.

To avoid association that may occur by chance when so many variables were evaluated, we considered an association to be significant if it was statistically significant $(p<0.05)$ in comparison with both controls, or at least for one of them with the second control showing an association in the same direction. Tables II-IX present data for all IBD patients together as well as for UC and CD patients separately.

\section{Results}

Table I shows the demographic characteristics of the study population and type and site of illness. Eighty seven patients with recent onset IBD were investigated as well as 76 population and 68 clinic controls. Each patient had at least one control and most $(83 \%)$ had two matched controls. There were 85 controls for the 54 patients with UC and 59 controls for the 33 patients with CD.

Tables II-IX summarise the main dietary findings expressed as odds ratios (OR) for IBD associated with three levels of intake of specific nutrients compared with each type of control

TABLE I Demographic characteristics

\begin{tabular}{|c|c|c|c|c|c|}
\hline & \multicolumn{3}{|l|}{ Patients } & \multicolumn{2}{|l|}{ Controls } \\
\hline & All & $U C$ & $C D$ & Population & Clinic \\
\hline $\begin{array}{l}\text { Number } \\
\text { Age (y) } \\
\% \text { Male }\end{array}$ & $\begin{array}{l}87 \\
29 \cdot 6(0 \cdot 80) \\
60\end{array}$ & $\begin{array}{l}54 \\
30 \cdot 14(1 \cdot 86) \\
55\end{array}$ & $\begin{array}{l}33 \\
29 \cdot 12(1 \cdot 99) \\
61\end{array}$ & $\begin{array}{l}76 \\
29 \cdot 4(1 \cdot 37) \\
63\end{array}$ & $\begin{array}{l}68 \\
29 \cdot 5(1 \cdot 38) \\
57\end{array}$ \\
\hline \multicolumn{6}{|l|}{ Community group } \\
\hline $\begin{array}{l}\text { Ashkenazi } \\
\text { Sephardi } \\
\text { Mixed }\end{array}$ & $\begin{array}{l}34 \\
32 \\
22\end{array}$ & $\begin{array}{l}24 \\
20 \\
10\end{array}$ & $\begin{array}{l}10 \\
12 \\
11\end{array}$ & $\begin{array}{l}31 \\
29 \\
16\end{array}$ & $\begin{array}{l}25 \\
27 \\
16\end{array}$ \\
\hline \multicolumn{6}{|l|}{ Disease location } \\
\hline $\begin{array}{l}\text { Small bowel (SB) only } \\
\text { Large bowel with or without SB }\end{array}$ & $\begin{array}{l}11 \\
76\end{array}$ & $\begin{array}{r}0 \\
54\end{array}$ & $\begin{array}{l}11 \\
22\end{array}$ & $\begin{array}{l}\text { NA } \\
\text { NA }\end{array}$ & $\begin{array}{l}\text { NA } \\
\text { NA }\end{array}$ \\
\hline
\end{tabular}


TABLE II Odds ratios (OR) for IBD by mean daily intake of carbohydrates (adjusted for energy intake)

\begin{tabular}{|c|c|c|c|c|c|c|c|c|c|c|}
\hline \multirow[b]{3}{*}{ Food source (g/day) } & \multicolumn{5}{|c|}{ Patients $\mathrm{v}$ clinic controls } & \multicolumn{5}{|c|}{ Patients $\mathrm{v}$ population controls } \\
\hline & \multicolumn{3}{|c|}{ Intake levels } & \multirow{2}{*}{$p^{\star \star}$} & \multirow{2}{*}{$C I^{\star \star \star}$} & \multicolumn{3}{|c|}{ Intake levels } & \multirow{2}{*}{$p^{\star \star}$} & \multirow{2}{*}{$C I^{\star \star \star}$} \\
\hline & Low & Medium & High & & & Low & Medium & High & & \\
\hline Total sugars ${ }^{\star}$ & $<107$ & $107-158$ & $>158$ & & & $<103$ & $103-163$ & $>163$ & & \\
\hline OR & & 1.8 & $3 \cdot 2$ & $0 \cdot 04$ & $1 \cdot 06-9 \cdot 61$ & 1 & $1 \cdot 13$ & $1 \cdot 27$ & 0.67 & $0 \cdot 42-3 \cdot 85$ \\
\hline Fructose & $<17$ & $17-38$ & $>38$ & & & $<17$ & $17-35$ & $>35$ & & \\
\hline OR & 1 & 0.73 & 0.53 & $0 \cdot 12$ & $0 \cdot 24-1 \cdot 18$ & 1 & 0.8 & $0 \cdot 6$ & 0.35 & $0.25-1.63$ \\
\hline Lactose & $<7$ & $7-18$ & $>18$ & & & $<6$ & $6-20$ & $>20$ & & \\
\hline OR & 1 & 1 & $0 \cdot 98$ & 0.97 & $0 \cdot 36-2 \cdot 7$ & 1 & 0.92 & 0.85 & $0 \cdot 70$ & $0.37-1.92$ \\
\hline Sucrose & $<54$ & $54-92$ & $>92$ & & & $<52$ & $52-93$ & $>93$ & & \\
\hline OR & 1 & $2 \cdot 3$ & $5 \cdot 3$ & $0 \cdot 00$ & $1 \cdot 62-17 \cdot 22$ & 1 & 1.7 & $2 \cdot 85$ & 0.03 & $1 \cdot 01-7 \cdot 58$ \\
\hline Fibres & $<18$ & $18-27$ & $>27$ & & & $<17$ & $17-26$ & $>26$ & & \\
\hline OR & 1 & 0.65 & $0 \cdot 43$ & $0 \cdot 20$ & $0 \cdot 12-1 \cdot 48$ & 1 & 0.87 & 0.77 & $0 \cdot 60$ & $0 \cdot 28-2 \cdot 07$ \\
\hline
\end{tabular}

All food quantities expressed in grams, unless otherwise indicated.

${ }^{\star} \mathrm{Di}$ - and monosaccharides; ${ }^{\star}$ linear trend; ${ }^{\star \star}{ }^{\star} 95 \%$ confidence interval, high $v$ low intake.

TABLE III Odds ratios (OR) for UC and CD by mean daily intake of carbohydrates (adjusted for energy intake)

\begin{tabular}{|c|c|c|c|c|c|c|c|c|}
\hline \multirow[b]{3}{*}{ Food source (g/day) } & \multicolumn{4}{|c|}{ UC patients $\mathrm{v}$ their controls } & \multicolumn{4}{|c|}{$C D$ patients $\mathrm{v}$ their controls } \\
\hline & \multicolumn{2}{|c|}{ OR for intake levels } & \multirow{2}{*}{$p^{\star \star}$} & \multirow{2}{*}{$C I^{\star \star \star}$} & \multicolumn{2}{|c|}{ OR for intake levels } & \multirow{2}{*}{$p^{\star \star}$} & \multirow{2}{*}{$C I^{\star \star \star}$} \\
\hline & Medium & High & & & Medium & High & & \\
\hline Total sugars ${ }^{\star}$ & 1.99 & 3.98 & 0.05 & $1 \cdot 02-15 \cdot 52$ & $1 \cdot 17$ & $1 \cdot 37$ & 0.63 & $0.37-5.09$ \\
\hline Fructose & 0.92 & 0.84 & $0 \cdot 70$ & $0.35-2.02$ & 0.65 & 0.42 & $0 \cdot 15$ & $0 \cdot 13-1 \cdot 36$ \\
\hline Sucrose & $2 \cdot 05$ & $4 \cdot 22$ & 0.02 & $1 \cdot 31-13 \cdot 60$ & 1.39 & 1.93 & 0.31 & $0 \cdot 54-6 \cdot 86$ \\
\hline Fibres & 0.90 & $0 \cdot 81$ & $0 \cdot 72$ & $0 \cdot 27-2 \cdot 47$ & 0.63 & $0 \cdot 40$ & $0 \cdot 21$ & $0 \cdot 10-1.65$ \\
\hline
\end{tabular}

All food quantities expressed in grams, unless otherwise indicated.

${ }^{\star} \mathrm{Di}$ - and monosaccharides; ${ }^{\star \star}$ linear trend; ${ }^{\star \star \star} 95 \%$ confidence interval, high $v$ low intake.

TABLE IV Odds ratios (OR) for IBD by mean daily intake of fat and protein (adjusted for energy intake)

\begin{tabular}{|c|c|c|c|c|c|c|c|c|c|c|}
\hline \multirow[b]{3}{*}{ Food source (g/day) } & \multicolumn{5}{|c|}{ Patients v clinic controls } & \multicolumn{5}{|c|}{ Patients v population controls } \\
\hline & \multicolumn{3}{|c|}{ Intake level } & \multirow{2}{*}{$p^{\star}$} & \multirow{2}{*}{$C I^{\star \star}$} & \multicolumn{3}{|c|}{ Intake level } & \multirow{2}{*}{$p^{\star}$} & \multirow{2}{*}{$C I^{\star \star}$} \\
\hline & Low & Medium & High & & & Low & Medium & High & & \\
\hline Fat - total & $<83$ & $83-119$ & $>119$ & & & $<80$ & $80-107$ & $>107$ & & \\
\hline OR & 1 & $1 \cdot 46$ & $2 \cdot 14$ & $0 \cdot 25$ & $0 \cdot 57-8 \cdot 00$ & 1 & $2 \cdot 30$ & $5 \cdot 31$ & 0.02 & $1 \cdot 35-20 \cdot 81$ \\
\hline Fat - animal & $<35$ & $35-54$ & $>54$ & & & $<33$ & $33-47$ & $>47$ & & \\
\hline OR & 1 & $1 \cdot 41$ & 1.99 & $0 \cdot 21$ & $0.68-5 \cdot 87$ & 1 & 1.90 & $3 \cdot 63$ & 0.02 & $0 \cdot 24-10 \cdot 62$ \\
\hline Fat - vegetable & $<16$ & $16-25$ & $>25$ & & & $<14$ & $14-24$ & $>24$ & & \\
\hline OR & 1 & 1.09 & $1 \cdot 20$ & $0 \cdot 70$ & $0 \cdot 45-3 \cdot 15$ & 1 & $1 \cdot 54$ & $2 \cdot 36$ & $0 \cdot 04$ & $1.04-5.35$ \\
\hline Saturated fat & $<30$ & $30-66$ & $>66$ & & & $<29$ & $29-53$ & $>53$ & & \\
\hline OR & 1 & $1 \cdot 29$ & 1.68 & $0 \cdot 27$ & $0 \cdot 66-4 \cdot 28$ & 1 & $1 \cdot 62$ & $2 \cdot 62$ & 0.05 & $1 \cdot 00-6 \cdot 80$ \\
\hline Monounsaturated fat & $<33$ & $33-55$ & $>55$ & & & $<29$ & $29-47$ & $>47$ & & \\
\hline OR & 1 & $1 \cdot 16$ & 1.35 & $0 \cdot 58$ & $0.46-3.99$ & 1 & 1.65 & $2 \cdot 72$ & 0.05 & $1 \cdot 01-7 \cdot 27$ \\
\hline Polyunsaturated fat & $<20$ & $20-30$ & $>30$ & & & $<19$ & $19-28$ & $>28$ & & \\
\hline OR & 1 & $1 \cdot 10$ & $1 \cdot 22$ & 0.77 & $0 \cdot 31-4 \cdot 81$ & 1 & $1 \cdot 82$ & $3 \cdot 30$ & 0.05 & $0.96-11.5$ \\
\hline Cholesterol (mg) & $<352$ & $352-563$ & $>563$ & & & $<345$ & $345-534$ & $>534$ & & \\
\hline OR & 1 & 1.91 & $3 \cdot 66$ & $0 \cdot 04$ & $1 \cdot 06-12 \cdot 62$ & 1 & $2 \cdot 12$ & $4 \cdot 47$ & $0 \cdot 06$ & $1.53-13 \cdot 01$ \\
\hline Protein - total & $<85$ & $85-120$ & $>120$ & & & $<41$ & $41-98$ & $>98$ & & \\
\hline OR & 1 & 1.09 & $1 \cdot 20$ & 0.78 & $0 \cdot 24-5 \cdot 94$ & 1 & 1.83 & $3 \cdot 35$ & $0 \cdot 12$ & $0.72-15.61$ \\
\hline
\end{tabular}

All food quantities expressed in grams, unless otherwise indicated

${ }^{\star}$ Linear trend; ${ }^{\star \star} 95 \%$ confidence interval, high $v$ low intake.

separately; 95\% confidence intervals are given. Results were also analysed against both controls together for all IBD patients (data not shown) and for UC and CD separately.

Table II presents data related to carbohydrate consumption. Sugar (di- and monosaccharide)

TABLE v Odds ratios (OR) for UC by mean daily intake of fat and protein (adjusted for energy intake)

\begin{tabular}{|c|c|c|c|c|}
\hline \multirow[b]{3}{*}{ Food source (g/day) } & \multicolumn{4}{|c|}{ UC patients $\mathrm{v}$ their controls } \\
\hline & \multicolumn{2}{|c|}{ OR for intake levels } & \multirow[t]{2}{*}{$p^{\star}$} & \multirow[t]{2}{*}{$C I^{\star \star}$} \\
\hline & Medium & High & & \\
\hline Fat - total & 1.98 & 3.94 & 0.07 & $0.92-16.89$ \\
\hline Fat - animal & $2 \cdot 02$ & $4 \cdot 09$ & 0.02 & $1.20-13.95$ \\
\hline Fat - vegetable & 1.65 & $2 \cdot 71$ & 0.04 & $1 \cdot 04-7 \cdot 09$ \\
\hline Saturated fat & 1.73 & 2.98 & 0.07 & $0 \cdot 92-9 \cdot 62$ \\
\hline Monounsaturated fat & 1.91 & $3 \cdot 66$ & 0.04 & $1.06-12 \cdot 58$ \\
\hline Polyunsaturated fat & 2.56 & $6 \cdot 54$ & 0.02 & $1.45-29 \cdot 68$ \\
\hline Cholesterol (mg) & $2 \cdot 14$ & $4 \cdot 57$ & 0.02 & $1 \cdot 28-16 \cdot 33$ \\
\hline Protein - total & 1.21 & $1 \cdot 47$ & 0.65 & $0 \cdot 28-7 \cdot 72$ \\
\hline
\end{tabular}

All food quantities expressed in grams, unless otherwise

${ }^{\star}$ Lindicated.
${ }^{\star}$ inear trend; ${ }^{\star} 95 \%$ confidence interval, high $v$ low intake. consumption was positively associated with risk for IBD. Significant results were obtained only when cases were compared with clinic controls, but the same trend was also observed against population controls. Lactose showed no association with IBD, while fructose intake was negatively associated with risk for IBD (NS). Sucrose consumption showed a consistent positive association with risk for IBD. This was significant against each of the control groups separately (clinic controls $\mathrm{p}=0.00$; population controls $p=0.03$; both controls together $\mathrm{p}<0.005)$. Consumption of starches did not show any consistent trend (data not shown), while fibre intake showed a negative association with risk for IBD against each of the control groups; this did not reach statistical significance. When data were analysed for patients with UC and CD separately (Table III), the same trends were observed. Due to the smaller numbers (particularly in CD) the significance of some findings was reduced. 
TABLE VI Odds ratios (OR) for IBD by mean daily intake of selected micronutrients (adjusted for energy intake)

\begin{tabular}{|c|c|c|c|c|c|c|c|c|c|c|}
\hline \multirow[b]{3}{*}{ Food source (mg/day) } & \multicolumn{5}{|c|}{ Patients v clinic controls } & \multicolumn{5}{|c|}{ Patients $\mathrm{v}$ population controls } \\
\hline & \multicolumn{3}{|c|}{ Intake level } & \multirow{2}{*}{$p^{\star}$} & \multirow{2}{*}{$C I^{\star \star}$} & \multicolumn{3}{|c|}{ Intake level } & \multirow{2}{*}{$p^{\star}$} & \multirow{2}{*}{$C I^{\star \star}$} \\
\hline & Low & Medium & High & & & Low & Medium & High & & \\
\hline Magnesium & $<293$ & $293-436$ & $>436$ & & & $<277$ & $277-426$ & $>426$ & & \\
\hline OR & & 0.93 & 0.87 & $0 \cdot 79$ & $0 \cdot 32-2 \cdot 38$ & & 0.54 & $0 \cdot 30$ & 0.04 & $0.09-0.96$ \\
\hline Calcium & $<582$ & $582-885$ & $>885$ & & & $<530$ & $530-885$ & $>885$ & & \\
\hline OR & & $1 \cdot 31$ & 1.69 & 0.32 & $0 \cdot 6-4 \cdot 8$ & & 1.33 & 1.77 & 0.31 & $0 \cdot 59-5 \cdot 25$ \\
\hline Potassium & $<3104$ & $3104-4033$ & $>4033$ & & & $<2924$ & $2924-3899$ & $>3899$ & & \\
\hline OR & 1 & 0.71 & 0.51 & $0 \cdot 21$ & $0 \cdot 17-1 \cdot 47$ & 1 & 0.77 & 0.59 & 0.38 & $0 \cdot 18-1.93$ \\
\hline Vitamin C & $<76$ & $76-139$ & $>139$ & & & $<71$ & $71-115$ & $>115$ & & \\
\hline OR & & 0.69 & 0.48 & 0.07 & $0 \cdot 71-1 \cdot 07$ & 1 & 0.82 & 0.68 & 0.43 & $0 \cdot 26-1 \cdot 76$ \\
\hline Carotene & $<2442$ & $2442-4106$ & $>4106$ & & & $<2159$ & $2159-4457$ & $>4457$ & & \\
\hline OR & & $1 \cdot 20$ & 1.43 & 0.40 & $0 \cdot 6-3 \cdot 40$ & & 1.04 & 1.09 & 0.80 & $0 \cdot 41-2 \cdot 85$ \\
\hline Retinol & $<377$ & $377-1224$ & $>1224$ & & & $<386$ & $386-1231$ & $>1231$ & & \\
\hline OR & 1 & $1 \cdot 82$ & $3 \cdot 32$ & 0.01 & $1 \cdot 31-8 \cdot 43$ & 1 & $1 \cdot 10$ & $1 \cdot 20$ & $0 \cdot 68$ & $0.49-2.95$ \\
\hline
\end{tabular}

${ }^{\star}$ Linear trend; ${ }^{\star \star} 95 \%$ confidence interval, high $v$ low intake.

TABLE VII Odds ratios (OR) for UC and CD by mean daily intake of selected micronutrients (adjusted for energy intake)

\begin{tabular}{|c|c|c|c|c|c|c|c|c|}
\hline \multirow[b]{3}{*}{ Food source (mg/day) } & \multicolumn{4}{|c|}{ UC patients $\mathrm{v}$ their controls } & \multicolumn{4}{|c|}{$C D$ patients $\mathrm{v}$ their controls } \\
\hline & \multicolumn{2}{|c|}{ OR for intake level } & \multirow{2}{*}{$p^{\star}$} & \multirow{2}{*}{$C I^{\star \star}$} & \multicolumn{2}{|c|}{ OR for intake level } & \multirow{2}{*}{$p^{\star}$} & \multirow{2}{*}{$C I^{\star \star}$} \\
\hline & Medium & High & & & Medium & High & & \\
\hline Magnesium & 0.60 & 0.36 & $0 \cdot 11$ & $0 \cdot 10-1 \cdot 25$ & 0.82 & 0.67 & 0.58 & $0 \cdot 16-2 \cdot 78$ \\
\hline Calcium & 1.54 & $2 \cdot 36$ & $0 \cdot 19$ & $0.65-8.59$ & $1 \cdot 15$ & 1.33 & 0.68 & $0 \cdot 35-5 \cdot 10$ \\
\hline Potassium & 1.09 & $1 \cdot 19$ & 0.80 & $0.31-4.58$ & 0.56 & 0.32 & 0.13 & $0.07-1.39$ \\
\hline Vitamin C & 0.87 & 0.76 & 0.55 & $0.30-1.91$ & 0.48 & 0.23 & 0.02 & $0.07-0.83$ \\
\hline Carotene & $1 \cdot 32$ & $1 \cdot 73$ & $0 \cdot 31$ & $0.60-4.98$ & 1.01 & 1.01 & 0.98 & $0.35-2.96$ \\
\hline Retinol & 1.49 & $2 \cdot 23$ & $0 \cdot 11$ & $0 \cdot 85-5 \cdot 87$ & $1 \cdot 21$ & 1.46 & 0.50 & $0 \cdot 49-4 \cdot 37$ \\
\hline
\end{tabular}

${ }^{\star}$ Linear trend; ${ }^{\star} 95 \%$ confidence interval, high $v$ low intake.

TABLE VIII Odds ratios (OR) for IBD by mean daily intake of selected foods and beverages (adjusted for energy intake)

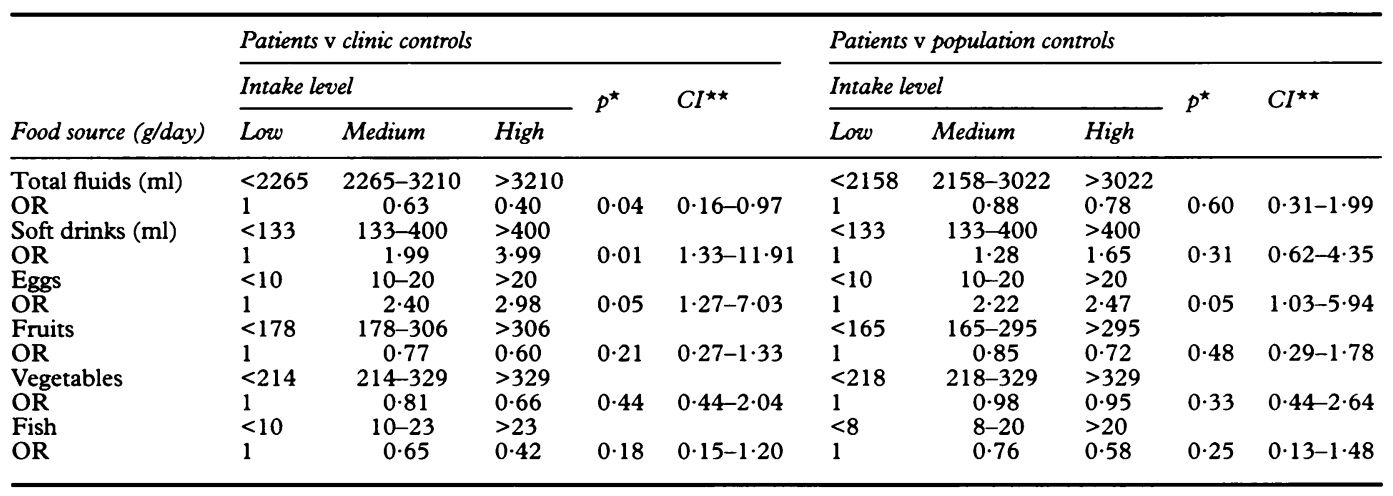

All food quantitites expressed in grams, unless otherwise indicated.

${ }^{\star}$ Linear trend; ${ }^{\star} 95 \%$ confidence interval, high $v$ low intake.

TABLE IX Odds ratios (OR) for UC and CD by mean daily intake of selected foods and beverages (adjusted for energy intake)

\begin{tabular}{|c|c|c|c|c|c|c|c|c|}
\hline \multirow[b]{3}{*}{ Food source (g/day) } & \multicolumn{4}{|c|}{ UC patients $\mathrm{v}$ their controls } & \multicolumn{4}{|c|}{$C D$ patients $\mathrm{v}$ their controls } \\
\hline & \multicolumn{2}{|c|}{ OR for intake level } & \multirow{2}{*}{$p^{\star}$} & \multirow{2}{*}{$C I^{\star \star}$} & \multicolumn{2}{|c|}{ OR for intake level } & \multirow{2}{*}{$p^{\star}$} & \multirow{2}{*}{$C I^{\star \star}$} \\
\hline & Medium & High & & & Medium & High & & \\
\hline $\begin{array}{l}\text { Total fluids (ml) } \\
\text { Soft drinks (ml) } \\
\text { Eggs } \\
\text { Fruits } \\
\text { Vegetables } \\
\text { Fish }\end{array}$ & $\begin{array}{l}0.67 \\
1 \cdot 84 \\
2 \cdot 98 \\
0 \cdot 80 \\
1 \cdot 13 \\
0 \cdot 81\end{array}$ & $\begin{array}{l}0 \cdot 45 \\
3 \cdot 39 \\
4 \cdot 45 \\
0.65 \\
1 \cdot 28 \\
0.65\end{array}$ & $\begin{array}{l}0 \cdot 12 \\
0.02 \\
0 \cdot 05 \\
0 \cdot 36 \\
0.57 \\
0.36\end{array}$ & $\begin{array}{l}0 \cdot 16-1 \cdot 22 \\
1 \cdot 25-9 \cdot 19 \\
1 \cdot 74-11 \cdot 34 \\
0 \cdot 25-1 \cdot 65 \\
0 \cdot 55-2 \cdot 95 \\
0 \cdot 26-1 \cdot 65\end{array}$ & $\begin{array}{l}0.80 \\
1.20 \\
1.88 \\
0.65 \\
0.57 \\
0.76\end{array}$ & $\begin{array}{l}0.68 \\
1.44 \\
1.76 \\
0.42 \\
0.32 \\
0.57\end{array}$ & $\begin{array}{l}0.49 \\
0.58 \\
0.66 \\
0 \cdot 15 \\
0.08 \\
0.39\end{array}$ & $\begin{array}{l}0.22-2 \cdot 06 \\
0.39-5.34 \\
0.58-5.39 \\
0.13-1.38 \\
0.09-1 \cdot 12 \\
0 \cdot 16-2.03\end{array}$ \\
\hline
\end{tabular}

All food quantitites expressed in grams, unless otherwise indicated.

${ }^{\star}$ Linear trend; ${ }^{\star} 95 \%$ confidence interval, high $v$ low intake.

Total energy consumption did not show any consistent association with risk for IBD (data not shown). Total protein consumption (particularly from animal origin), showed a non-significant positive association with risk for IBD (Table IV).
Fat consumption showed a marked significant association with risk for IBD. The same trend was noted against both types of controls. This increased risk existed with all types of fats (animal, vegetable, saturated, and unsaturated). The highest OR was noted for 
cholesterol consumption. This association was marked in patients with UC (Table V). The differences were minimal in patients with $C D$ except for animal fat and cholesterol which showed a positive association (NS).

Table VI shows data for selected micronutrients (mainly minerals and vitamins). Magnesium consumption showed a negative association with risk for IBD which was significant only against population controls. Patients consumed more calcium than the controls, but this difference was not significant. Potassium consumption was negatively associated with risk for IBD against both types of controls; however statistical significance was not achieved. An increased intake of vitamin C was negatively associated with risk for IBD compared with both controls, with borderline significance $(p=0 \cdot 07)$. Consumption of retinol was however significantly associated with risk for IBD. The consumption of other vitamins did not show any significant trend (data not shown). In patients with UC or CD (analysed separately) the results were similar except for potassium consumption in patients with UC which did not show a negative association.

Table VIII summarises intake of selected food items and beverages. Total fluid consumption (in liquids as well as in solid foods) showed a significant negative association with risk for IBD. This reached statistical significance only against clinic controls. Soft drinks (most of which contain sugar) showed a positive association with IBD which was significant against clinic controls. The consumption of eggs showed a positive trend for IBD compared with controls $(p<0.05)$, while the consumption of fruit, vegetables, and fish showed a negative association. All the above associations were not statistically significant. Table IX shows data for patients with UC and $C D$. The negative association with increasing fluid intake was found in patients with both diseases (NS), as was the positive association with the consumption of soft drinks. It reached statistical significance only in patients with UC. Fruit and fish consumption showed the same association in patients with UC and $C D$, while vegetable consumption did not show a negative association with risk for UC. Egg consumption was positively associated only with UC. All other analysed foods showed no consistent trend or correlation with the risk for IBD, UC, or CD.

\section{Discussion}

The study of associations between nutrition and disease presents methodological problems, especially when studying the long term effects of a previous diet on a current disease process. ${ }^{25} 29$ Cross-sectional studies evaluating the current diet are of little value as nutritional habits are already influenced by the illness itself. Our quantified dietary history questionnaire, allowing investigations of diet prior to illness is, under these circumstances, the most reliable tool.

Two further conditions have to be met: the dietary interview should be conducted as close as possible to the initial diagnosis or, prefer- ably, to disease onset; and the method of subject selection and data analysis is paramount.

In the analysis of dietary data, a comparison of mean intakes in the test and control groups constitutes inappropriate methodology. Arithmetic means are heavily influenced by individual variations. As has been pointed out in several nutritional studies, ${ }^{23-25}$ including IBD, ${ }^{7}$ the more appropriate methodology for dietary investigations is the odds ratio.

The choice of controls is also crucial. Hospital controls have been used in many studies; however, randomly chosen population controls are preferable. Having two types of control may protect against bias associated with a specific type of control. Matching by age and sex is appropriate; however, socioeconomic and ethnic factors may be much more important in dietary studies.

With these considerations in mind, we reviewed published nutritional studies on patients with IBD. Most studies ${ }^{14} 153031$ questioned patients with chronic established disease. Under these conditions, even when the questions refer to the pre-illness diet, the time factor markedly decreases the reliability of results. Only a few studies questioned patients close to the time of diagnosis, and none of them used the odds ratio methodology. ${ }^{11-13}$ 18-20

Several studies used postal self-reported questionnaires $^{121419}$ instead of a direct interview by an experienced dietician. Other studies confined the dietary questions to a few selected items such as sugar added to drinks, breakfast cereals, milk feeding in infancy, etc, and did not conduct a full dietary interview. ${ }^{12-14} 19$

Thus, to the best of our knowledge, the present study is the only one combining the investigation of patients with recent onset of disease with a full analysis of the pre-illness diet using conditional logistic analysis. Furthermore, randomly chosen population controls were used in addition to clinic controls, and the matching included socioeconomic and demographic characteristics. All the above strengthen the validity of our results.

Previous studies of patients with chronic IBD have demonstrated the following findings. Sugar intake was increased in patients with IBD compared with controls in three large European studies. ${ }^{14} 1530$ This trend reached statistical significance in patients with $\mathrm{CD}^{14} 30$ and sometimes also in patients with UC. ${ }^{15}$ Fruit, vegetable, and fibre consumption was found to be significantly lower in patients with IBD. ${ }^{15}{ }^{30} \mathrm{~A}$ Japanese study ${ }^{31}$ compared patients with UC with controls and found decreased vegetable and fruit consumption (NS), while the intake of a Western type diet increased the risk for UC. Studies of patients with recent diagnosis of IBD analysed only the mean intakes of nutrients of patients in comparison to controls. They found an increase in sugar intake in patients with $C D$ compared with controls. ${ }^{11-131920}$ This difference was not found in the three studies in which patients with UC were evaluated. ${ }^{13} 1819$ Fibre consumption in patients with $\mathrm{CD}$ was low in two 
of these studies ${ }^{11} 19$ and high in one. ${ }^{20}$ In another study, ${ }^{13}$ the increased sugar intake in patients with $\mathrm{CD}$ was found only in patients with long standing disease, and it was concluded that it was a result rather than a cause of the disease.

In our study, we confirmed the increased sucrose consumption, and showed that this antedated disease. This was not a general phenomenon related to carbohydrate or even to other disaccharide or monosaccharide consumption. Thus, the ingestion of fructose, which is found more in fruits, was decreased in the pre-illness diet of patients with IBD compared with controls. Moreover, no difference was found in lactose consumption or in polysaccharide consumption. The deleterious effect was confined to sucrose only.

The main new finding in our study was related to the increased fat consumption in the pre-illness period of patients mainly with UC. This increased consumption was found with all types of fats (animal fat, vegetable fat, saturated as well as unsaturated fat, and cholesterol). This increased risk for UC in subjects consuming a high fat diet prior to the onset of disease has, to the best of our knowledge, not been previously reported. Fat is a risk factor in many diseases; its mechanism of action in $\mathrm{UC} / \mathrm{IBD}$ is at present unknown. An increased use of chemically modified fats including suppositories was reported in patients with CD. ${ }^{32} 33$

Protein consumption did not seem to play a role. A decreased risk for IBD with increasing water as well as potassium (in CD only), magnesium, and vitamin $C$ consumption was observed. There may be a certain interplay in these findings. A high sucrose consumption is usually associated with an increased consumption of refined food and a decreased fruit and vegetable intake. In contrast, fructose, potassium, magnesium, vitamin $\mathrm{C}$, fibre, and also water are found particularly in fruits and vegetables. Thus, as a generalisation, these findings point to low residue, refined foods as a risk factor, and a high fruit, vegetable, and fibre diet as a protective factor. This generalisation may, however, be too facile as, for example, the consumption of vitamin A precursors was positively associated with risk for IBD while other vitamins such as the various $B$ vitamins showed no clear association. It is, therefore, equally possible that the effects of fructose, vitamins $C$ and $K$, magnesium, and fibre are not exclusively due to their presence in fruits and vegetables. Water consumption can be considered in two ways. One is that of a higher consumption of fruits and vegetables which contain mostly water. The other is that the concentration of any ingested deleterious agent will be diluted by a high fluid intake. Such an effect has been demonstrated in experimental carcinogenesis. ${ }^{34}$ Interestingly, the effect of calcium tended to be positively associated with the risk for IBD even though this did not reach a level of statistical significance. This is unlike the situation in colon carcinoma where calcium has been found to be protective. On the other hand, calcium and magnesium have often been found to have opposing effects.
In previous studies an increased sucrose consumption was found only in patients with CD. Subsequently, it was shown in a few studies to be true for UC. Most of these studies related to the post-illness diet. In our study, sucrose consumption prior to disease showed a similar trend in UC and CD.

Sugar and smoking may confound one another. Smoking habits have been recorded in our study population and, in contrast to most other studies, we did not find an excess of smoking in CD and paucity of smokers in UC. No significant differences in smoking were demonstrated between patients and controls in our study. ${ }^{35}$ This may be a situation particular to Jewish patients at least in our area. ${ }^{35}$

Most of our findings relating to the preillness diet were similar in UC and CD. The exceptions were that intake of vegetables, potassium, eggs, and fat was high in UC. In $\mathrm{CD}$ only animal fat and cholesterol (which are considered to be the most deleterious) were positively but not significantly associated with the risk for disease.

Our findings suggest that dietary factors are important in the development of IBD. These factors may have a primary effect in predisposed subjects, or may modulate the effect of other as yet unidentified environmental factors. If and when our findings are confirmed by other investigators, they may be considered for practical application. For instance, in a family with one or more first degree relatives with IBD, a "prudent diet" may be recommended for uninvolved members of the family, particularly the children. This may, in the future, apply mainly to those family members shown to be genetically predisposed to IBD.

Part of this study is included in the MSc thesis of I Klein, Tel Aviv University, Tel Aviv, Israel. The authors wish to express their appreciation to the numerous gastroenterologists in the Tel Aviv area who referred their newly diagnosed patients with IBD for this study.

1 Tysk C, Lindberg C, Jarnerot G, Floderus-Myrhed B. Ulcerative colitis and Crohn's disease in an unselected population of monozygotic and dizygotic twins. A study population of monozygotic and dizygotic twins. A study of heritabili.

2 Monsen U, Brostrom O, Nordenvall B, Sorstad J, Hellers G. Prevalence of inflammatory bowel disease among relatives of patients with ulcerative colitis. Scand $\mathcal{F}$ Gastroenterol 1987; 22: 214-8.

3 Mayberry JF, Rhodes J, Newcombe RG. Familial prevalence of inflammatory bowel disease in relatives with Crohn's disease. $B M \mathcal{F} 1980 ; 1: 84$.

4 Nakajima A, Matsuhashi N, Kodama T, Nazaki Y, Takazoe M, Kimura A. HLA linked susceptibility and resistance gene in Crohn's disease. Gastroenterology 1995; 109: $1462-7$.

5 Gilat T, Langman MJS, Rozen P. Environmental factors in inflammatory bowel disease. Front Gastrointest Res 1986; 11: $158-76$

6 Gilat T, Hacohen D, Lilos P, Langman MJS. Childhood factors in ulcerative colitis and Crohn's disease. An international cooperative study. Scand $\mathcal{f}$ Gastroenterol 1987; 22: 1009-24

7 Person PG, Ahlbom A, Hellers G. Crohn's disease and ulcerative colitis: a review of dietary studies with emphasis on methodologic aspects. Scand f Gastroenterol 1987; 22: $385-9$.

8 Harries $\mathrm{AD}$, Baird A, Rhodes J. Non-smoking: a feature of ulcerative colitis. $B M \mathcal{F}$ 1982; 284: 706 .

9 Jick H, Walker AM. Cigarette smoking and ulcerative colitis. N Engl f Med 1983; 308: 261-3.

10 Tobin MV, Logan RFA, Langman MJS, McConnell RB, Gilmore IT. Cigarette smoking and inflammatory bowel Gilmore IT. Cigarette smoking and inflam
disease. Gastroenterology 1987; 93: 316-21.

11 Thornton JR, Emmet PM, Heaton KW. Diet and Crohn's disease: characteristics of the preillness diet. BMF 1979; 2: $762-4$. 
12 Mayberry JF, Rhodes J, Newcombe RG. Increased sugar consumption in Crohn's disease. Digestion 1980; 20: 323-6.

13 Jarnerot G, Jarnmark I, Nilsson K. Consumption of refined sugar by patients with Crohn's disease, ulcerative colitis, sugar by patients with Crohn's disease, ulcerative colitis,
or irritable bowel syndrome. Scand $\mathcal{F}$ Gastroenterol 1983; 18: $999-1002$.

14 Mayberry JF, Rhodes J, Allan R, Newcombe RG, Regan GM, Chamberlain LM, et al. Diet in Crohn's disease. Dig Dis Sci 1981; 26: 444-8

15 Panza E, Franceschi S, La Vecchia C, Parazzini F, Petrillo M, De-Carli A, et al. Dietary factors in the aetiology of inflammatory bowel disease. Ital 7 Gastroenterol 1987; 19: 205-9.

16 Sandler RS, Golden AL. Epidemiology of Crohn's disease. 7 Clin Gastroenterol 1986; 8: 160-5.

17 Mayberry JF, Rhodes J. Epidemiologic aspects of Crohn's disease: a review of the literature. Gut 1984; 25: 886-99.

18 Thornton JR, Emmet PM, Heaton KW. Diet and ulcerative colitis. BMF 1980; 280: 293-4.

19 Thornton JR, Emett PM, Heaton KW. Smoking, sugar and inflammatory bowel disease. BMF 1985; 290: 1786-7.

20 Kasper H, Sommer H. Dietary fiber and nutrient intake in Crohn's disease. Am $\mathcal{F}$ Clin Nutr 1979; 32: 1898-901.

21 Modan B, Lubin F, Barell V, Greenberg RA, Modan M, Graham S. The role of starches in the etiology of gastric cancer. Cancer 1974; 34: 2087-92.

22 Modan B, Lubin F, Barell V, Greenberg RA, Modan M, Graham S. Low fiber intake as an etiologic factor in cancer of the colon. 7 Natl Cancer Inst $1975 ; 55: 15-8$.

23 Lubin F, Wax Y, Modan B. The role of fat, animal protein and dietary fiber in breast cancer etiology - A caseand dietary fiber in breast cancer etiology - A

24 Lubin F, Ruder A, Wax Y, Modan B. Overweight and changes in weight through adult life in breast cancer: a case-control study. Am $\mathcal{f}$ Epidemiol 1985; 122: 579-88.
25 Lubin F, Boeing H, Rozen P. Design and background of the Tel-Aviv - Heidelberg dietary study of colonic adenoma patients and calcium intervention trial. Front Gastrointest Res 1991; 18: 74-87.

26 Guggenheim YK, Kaufman NA, Reshef A. Composition of foods. 6th ed. Jerusalem: Magens Press, The Hebrew foods. 6th ed. Jerus
University, 1991.

27 Paul AA, Southgate DAT. In: McCance and Widowson, eds. The composition of foods. 4th ed. London: Elsevier, 1978

28 Breslow NE, Day NE. Statistical methods in cancer research. In: The analysis of case control studies. Lyon: IARC, 1980; 32: 202-5.

29 Rothman KJ. Modern epidemiology. Boston, Toronto: Little Brown and Company, 1986; 327-31.

30 Persson PG, Ahlbom A, Hellers G. Diet and inflammatory bowel disease: a case-control study. Epidemiology 1992; 3: $47-52$.

31 Kono S. Dietary and other risk factors of ulcerative colitis. A case-control study in Japan. $\mathcal{F}$ Clin Gastroenterol 1994; 19: $166-71$.

32 Emerit J, Congy F, Loeper J, Verlingue D. Incidence de la consommation de margarine sur la frequence de la maladie de Crohn. Presse Med 1983; 43: 2767-8.

33 Guthy E, Schroder A, Paranype S. Fettverzehr, Ausser-Haus-Verpflegung und Zapfchenverbrauch bei Crohn-Patienten. Dtsch Med Wochenschr 1983; 108: 1719-20.

34 Lubin F, Rozen P, Arieli B, Farbstein M, Kaanni E, Bat L, et al. Nutritional and lifestyle habits and water-fiber interaction in colorectal adenoma etiology. Cancer Epidemiol Biomarkers Prev 1997; 6: 79-85.

Epidemiol Biomarkers Prev 1997; 6: 79-85.
35 Reif S, Klein F, Arber N, Gilat T. Lack of association between smoking and inflammatory bowel disease in Jewish patients in Israel. Gastroenterology 1995: 108: 1683-7. 